\title{
Safe obstetric anesthesia practice-COVID-19
}

\author{
Sunil Thakur ${ }^{1}$, Sushruti Kaushal ${ }^{2 *}$, Harpreet Kaur ${ }^{3}$, Asmita Kaundal ${ }^{2}$
}

\author{
${ }^{1}$ Department of Anesthesiology, Dr. RK Govt Medical College, Hamirpur, Himachal Pradesh, India \\ ${ }^{2}$ Department of Obstetrics and Gynaaecology, All India Institute of Medical Sciences, Bilaspur, Himachal Pradesh, India
}

Received: 13 October 2021

Accepted: 09 November 2021

\section{*Correspondence:}

Dr. Sushruti Kaushal,

E-mail: sushrutikaushal@gmail.com

Copyright: (C) the author(s), publisher and licensee Medip Academy. This is an open-access article distributed under the terms of the Creative Commons Attribution Non-Commercial License, which permits unrestricted non-commercial use, distribution, and reproduction in any medium, provided the original work is properly cited.

\begin{abstract}
COVID-19 or Corona virus disease 2019 was declared a pandemic by the WHO on $11^{\text {th }}$ March 2020. Cesarean delivery is a commonly performed major surgery around the world. Important considerations while performing a cesarean section on a confirmed or suspected case of COVID19 are safety of the woman and the fetus; and prevention of transmission of SARS-CoV2 infection to the fetus as well as the healthcare providers. Woman and her birthing partner should be screened for the symptoms of COVID-19 before scheduled admission. Cesarean section in a woman suffering from COVID-19 should be managed by a multidisciplinary team consisting of anesthetists, obstetricians, labor and delivery nurses, neonatologist, critical care experts and infectious disease specialists, all members working in tandem with each other. General changes in workflow, reorganization of obstetric anesthesia services and proper use of personal protective equipment (PPE) are required for safe delivery of obstetric anesthesia during the COVID pandemic. Regional anesthesia is the preferred method of anesthesia for cesarean delivery. Using regional anesthesia reduces the need of aerosol generating procedures and avoids the use of mechanical ventilation. It also decreases the possibility of exacerbating the respiratory complications due to intubation. General anesthesia is recommended when a COVID-19 parturient presents with desaturation (oxygen saturation $\leq 93 \%$ ) for emergency cesarean delivery. Use of mechanical barriers around patient's head during intubation and extubation might reduce exposure. The patient should be allowed to remain in the operating room itself till sufficiently recovered to be shifted directly to the isolation room.
\end{abstract}

Keywords: Obstetric, Anesthesia, Cesarean, Fetus, COVID-19

\section{INTRODUCTION}

COVID-19 or Corona virus disease 2019 was first reported in December 2019 at Wuhan, China and within 3 months of its onset, it had crippled the whole world. It was declared a pandemic by the WHO on $11^{\text {th }}$ March 2020. Since then, almost all the countries have been struggling with repeated waves of infection. Numerous vaccines based on different technologies have been developed but the end of the pandemic is still beyond the horizon. Even after the pandemic ends, virus is likely to keep circulating and possibly causing local outbreaks. Compared to their non-pregnant peers, infected pregnant women have been shown to develop more serious illness and needing more intensive care unit (ICU) admissions once symptomatic.
Pregnancy is a state of immune-suppression and pregnant women have previously been documented to be at high risk due to various infectious diseases including respiratory infections like influenza, severe acute respiratory syndrome (SARS) and middle east respiratory syndrome (MERS). Sufficient evidence has now accumulated to suggest that pregnant women and their fetuses are at high risk of complications due to COVID-19. ${ }^{1}$ A significant number of pregnant women require cesarean section for the termination of their pregnancies. Although there has been a tendency towards postponement of elective and non-emergent surgeries during the pandemic, cesarean sections remain a commonly performed unavoidable major surgery around the world. Keeping this in mind, safe anesthesia practice during labor and delivery is paramount 
to ensure the safety of the parturient, the neonate and the concerned anesthesia and obstetric teams.

\section{PREGNANCY AND COVID-19: IMPORTANT ASPECTS DURING LABOR AND DELIVERY}

As pregnancy is an immuno-compromised state, there is impaired ability to fight various infections, including viral infections like SARS-CoV2 infection. Based on our experience with previous corona virus outbreaks-MERS and SARS, it is only expected for SARS-CoV2 infection to be of higher severity in pregnant females. A review of laboratory confirmed cases of COVID-19 during pregnancy, conducted in the US, reported increased risk of hospitalization, ICU admission, mechanical ventilation, extracorporeal membranous oxygenation (ECMO) and death, when compared with non-pregnant adults. ${ }^{2}$

Although the risk of severe disease in pregnancy is higher than in general population, the absolute risk of severe disease remains low. Majority of pregnant women $(86 \%)$ develop mild disease, $9 \%$ have severe disease and $5 \%$ develop critical illness. ${ }^{3}$ Third trimester of pregnancy and post-partum are the 'at-risk' periods and most of the severe cases have been reported during these time-frames. ${ }^{4}$

Pregnant women with co-morbidities like diabetes, hypertension and obesity are at an even higher risk of severe disease due to COVID-19. These women with comorbidities are also at higher risk of anesthesia related complications, making them particularly vulnerable during a surgical procedure like a cesarean section.

SARS-CoV2 infection carries a risk of thrombo-embolic complications, a risk that could be further augmented by pregnancy and cesarean section. Based on this consideration, Royal college of obstetricians and gynaecologists (RCOG) has emphasized the importance of appropriate thromboprophylaxis during pregnancy and after cesarean section in women who are infected with COVID-19 during pregnancy.

Important considerations while performing a cesarean section on a confirmed or suspected case of COVID19 are safety of the woman and the fetus; and prevention of transmission of SARS-CoV2 infection to the fetus as well as the healthcare providers. Furthermore, precautions are needed in all women undergoing delivery and cesarean to prevent them from getting infected during hospital visit or hospital admission.

\section{GENERAL CONSIDERATIONS}

\section{Pre-hospital considerations}

Woman and her birthing partner should be screened for the symptoms of COVID-19 (fever, cough, respiratory symptoms) on the day before a scheduled admission. If the woman reports presence of any of the above symptoms, admission can be rescheduled for 2-3 days if feasible, to allow for results of testing for SARS-CoV2 infection. For COVID-19 positive women with mild to moderate symptoms, consideration should be given to the fact that severity of disease often peaks in the second week. It is optimal to plan delivery or cesarean keeping this in mind. ${ }^{5}$

\section{Pre-operative considerations}

\section{General changes to routine workflow}

All women and their birthing attendants should be screened at the time of admission for symptoms suggestive of SARS-CoV2 infection. If the woman tests positive on screening, woman should be directed towards appropriate testing or medical care as indicated by her clinical condition. If a birthing attendant shows symptoms of COVID-19 during screening, he/she should not be allowed to accompany the parturient woman and should be directed towards appropriate testing and medical care. ${ }^{5}$

Where feasible, a policy for universal testing of all women getting admitted for delivery/cesarean can be made. This is because a large number of SARS-CoV2 infected people remain asymptomatic while continuing to spread the disease. ${ }^{3}$

A special room, with facility for delivery/cesarean, should be reserved for screen-positive women waiting for triage. Under ideal circumstances, there should be demarcated areas for non-infected, suspect and infected women with separate passageways. Each area should have its own operation theatre and ICU. If space constraints don't allow for this segregation, all attempts should be made to avoid women in different categories coming in proximity of each other.

\section{Reorganizing obstetric anesthesia services}

Cesarean section in a woman suffering from COVID-19 should be managed by a multidisciplinary team consisting of anesthetists, obstetricians, labor and delivery nurses, neonatologist, critical care experts and infectious disease specialists; all members working in tandem with each other. ${ }^{6}$

There should be well established protocols regarding admission, treatment and transport of COVID-19 patients to streamline the management of COVID-19 patients.

Lines of communication between the obstetric and anesthesia teams should be well established, especially for emergency cesarean deliveries. Having a quick response team consisting of anesthetists, obstetricians and supportive staff can avoid delay in conducting an emergency cesarean delivery. Early information about a possible cesarean in a suspect/confirmed COVID-19 patient will enable the operating room team to be prepared for the surgery, will save precious time and will decrease the chances of error and disease transmission. ${ }^{6}$ 
Dedicated operating rooms and isolation rooms should be identified and restructured according to regulatory guidelines to prevent spread of airborne infections. Ideal scenario is to have rooms with negative pressure to prevent outflow of air.

Practicing appropriate donning and doffing practices, simulation trainings and considering transfer arrangements for infected women is absolutely necessary to prevent spread of infection from the infected patient. ${ }^{7}$

\section{Personal protective equipment}

Personal protective equipment (PPE), along with personal isolation and hygiene remain the cornerstones in our fight with COVID-19. Patient and her birthing attendant should wear surgical masks throughout the duration of their hospital stay. ${ }^{8}$ All healthcare workers should observe noncontact precautions, droplet precautions or airborne precautions depending on the type of exposure. ${ }^{9}$

Using a checklist and, if available, an observer at the time of donning and doffing of PPE decreases chances of error.

Obstetric anesthetists' association, Royal college of anesthetists and other associations issued a joint statement and recommended the use of apron, fluid resistant disposable gown, sterile gloves, eye protection and fluid resisting surgical mask during a cesarean delivery under neuraxial block. For a cesarean done under general anesthesia, filtering face piece respirator-3 should replace a surgical mask. CDC has indicated that N95 masks are as effective as powered respirators and should be used in case of non-availability of powered respirators. ${ }^{9,10}$

\section{INTRAOPERATIVE MANAGEMENT}

A few important considerations to ensure success and to increase safety are: Admission of the patient in isolation room preferably with negative pressure, arrangement of safe transfer from ward to operating room, minimizing the number of people in operating room, creating COVID-19 kits with all equipment including drugs to decrease traffic, discussing the plan for difficult intubation and having equipment required for same like supraglottic airway and surgical airway kit immediately available, detailed recordkeeping, preferably digital, having post-operative care strategy ready in advance, anticipating needs to avoid emergency situations and timely decision-making to avoid delays and using non-verbal communication and sign language. ${ }^{11}$

\section{Type of anesthesia}

\section{Neuraxial block}

Regional anaesthesia is the preferred method of anaesthesia for cesarean delivery as it is considered safer. Using regional anaesthesia reduces the need of aerosol generating procedures and avoids the use of mechanical ventilation. It also decreases the possibility of exacerbating the respiratory complications due to intubation.

Spinal anesthesia, combined spinal and epidural anesthesia or extension of labor epidural can be used depending on operator preference. Rapid sequence spinal anesthesia is an option for emergency cesarean delivery. A single shot spinal with a $25-26 \mathrm{G}$ needle should be preferred as it carries the least chance of neuraxial seeding with the virus. ${ }^{6}$ Morphine may be added to bupivacaine under cover of prophylactic anti-emetics. Use of excessive intraoperative fluids should be avoided.

There have been reports of presence of thrombocytopenia in women with severe COVID-19. It is advisable to have a platelet count before regional anesthesia and to proceed with neuraxial block only if the count is more than 70$80,000 \times 10^{6} / \mathrm{L}$.

\section{General anesthesia}

General anesthesia during a cesarean delivery is recommended when a COVID-19 parturient presents with desaturation (oxygen saturation $\leq 93 \%$ ) for emergency cesarean delivery. Some procedures in general anesthesia are aerosol generating, so additional precautions are required. An intubation checklist should be used with closed loop communication because PPE makes communication difficult. Preoxygenation should occur with a circuit extension and a HEPA filter at the patient side of the circuit. ${ }^{12}$ High flow nasal oxygen is not recommended for preoxygenation. Rapid sequence induction should be done with a cuffed endotracheal tube. Use of video-laryngoscopy is preferable, if available. In case of a difficult intubation, second-generation supraglottic airway can be used. It is recommended to wear double pair of gloves and to discard the outer pair after securing the endo-tracheal tube. ${ }^{7}$ This is because the outer gloves are likely to get respiratory secretions during intubation. Position of the endo-tracheal tube should be checked by chest wall expansion and end tidal $\mathrm{CO}_{2}$, to avoid auscultation.

Use of mechanical barriers around patient's head during intubation and extubation might reduce exposure. Plastic intubation cubes/boxes, placing clear plastic sheet over patient while intubating and draping plastic sheet over Mayo stand have been tried. However, there is no guidance yet whether these methods are effective or if any technique is superior to the other. ${ }^{13}$

Presence of systemic complications of COVID-19, like renal failure or disseminated intravascular coagulation (DIC) may warrant the use of invasive monitoring during cesarean.

Extubation after surgery should be performed with the same precautions as intubation. Patients tend to be more agitated at the time of extubation and there could be more chances of viral dissemination from coughing than during 
intubation. Number of people in the operating room should be minimized during this time. ${ }^{7}$

\section{POST-OPERATIVE MANAGEMENT}

Transferring the COVID-19 patient to post-anesthesia care unit may risk exposure of other patients to SARS-CoV2 virus. The patient should be allowed to remain in the operating room itself till sufficiently recovered to be shifted directly to the isolation room. ${ }^{14}$

Standard post-operative care must be given with emphasis on pain-relief, pulse oximetry, judicious use of fluids and appropriate precautions in handling the neonate. Adequate analgesia is important because tachypnea associated with pain might exacerbate hypoxia. ${ }^{6}$ There is no evidence to suggest that non-steroidal anti-inflammatory drugs (NSAIDS) should not be given in these patients. ${ }^{15}$

There is evidence of hypercoagulability in patients with COVID-19. Pregnancy and postpartum have been established as hypercoagulable states. Additive effect of these factors in parturient women infected with SARS$\mathrm{CoV} 2$ virus can make these women particularly vulnerable to have thromboembolism. All women undergoing cesarean section should be given thromboprophylaxis, duration of which is guided by thromboprophylaxis guidelines.

\section{CONCLUSION}

Most of the pregnant women with SARS-CoV2 infection will have mild disease while a few will develop disease life-threatening severity. Our healthcare system must be prepared to screen all the patients, follow adequate precautions to prevent spread of virus from the asymptomatic women and provide standard of care to women suffering from severe/ critical disease. Handhygiene, universal use of face mask and social-distancing should be ensured. Forming institutional protocols and checklists for anesthesia services and adequate training of staff including simulation trainings are required to ensure safety of the patient as well as the healthcare worker, especially during this pandemic.

\section{Funding: No funding sources \\ Conflict of interest: None declared \\ Ethical approval: Not required}

\section{REFERENCES}

1. CDC. COVID-19 and Your Health. Centers for Disease Control and Prevention. 2020. Available at: https://www.cdc.gov/coronavirus/2019-ncov/needextra-precautions/pregnant-people.html. Accessed on 2021 Jun 2.

2. Zambrano LD, Ellington SR, Strid P, Galang RR, Oduyebo T, Tong VT et al. Characteristics of women of reproductive age with laboratory-confirmed SARSCoV-2 infection by pregnancy status-United States,
January 22-October 3, 2020. MMWR Morb Mortal Wkly Rep. 2020;1641-7.

3. Breslin N, Baptiste C, Gyamfi-Bannerman C. Coronavirus disease 2019, infection among asymptomatic and symptomatic pregnant women: two weeks of confirmed presentations to an affiliated pair of New York City hospitals. Am J Obstet Gynecol MFM. 2020;(2):100-18.

4. Royal College of Obstetricians and Gynaecologists. Coronavirus (COVID-19) infection in pregnancy. London: RCOG. 2020.

5. Boelig RC. Tracy Manuck, Oliver EA, Mascio DD, Saccone G, Bellussi F, Berghella V. Labor and delivery guidance for COVID-19. AJOG MFM. 2020;1-9.

6. Ganesh V, Bhatia R, Trikha A. COVID-19: Considerations for Obstetric Anesthesia and Analgesia. J Obstet Anaesth Crit Care. 2020;10:2.

7. Davenport G, Eddleston J, Grocott M. Clinical Guide for the management of Critical care adults with COVID-19 during Corona virus Pandemic. 2020.

8. Harenberg J, Church R, Tubug T. Anesthesia Considerations of a Pregnant Woman With COVID19 Undergoing Cesarean Delivery: A Case Report. AANA. 2020;47-53.

9. Lucas N, Bamber J, Donald F, Platt F. clinical guidelines-PPE for COVID-19. 2020; Available at: https://www.oaa-

anaes.ac.uk/assets/_managed/cms/files/Clinical\%20 Guidelines/OAA-PPE-infographic_11.04.20.pdf. Accessed on 2021 Jun 2.

10. Centers for Disease Control and Prevention. Interim infection prevention and control recom- mendations for patients with suspected or confirmed coronavirus disease 2019 (COVID- 19) in healthcare settings. Available at: https://www.cdc.gov/coronavirus/2019ncov/infectioncontrol/controlrecommendations.html. Accessed March 23, 2021.

11. An Update on the Perioperative Considerations for COVID-19 Severe Acute Respiratory Syndrome Coronavirus-2 (SARS-CoV-2). Anesthesia Patient Safety Foundation. Available at: https://www.apsf.org/article/an-update-on-theperioperative-considerations-for-covid-19-severeacute-respiratory-syndrome-coronavirus-2-sars-cov2/. Accessed on 2021 Jun 16.

12. Miller E, Leffert L, Landau R. Society for Obsteric Anestesia and Perinatology. Interim Con-siderations for Obstetric Anaesthesia Care related to COVID19. Available at: http://soap.org. Accessed on 2021 Jun 2.

13. COVID-19 and Anesthesia FAQ. Anesthesia Patient Safety Foundation. Available at: https://www.apsf.org/covid-19-and-anesthesia-faq/. Accessed on 2021 Jun 16.

14. Ashokka B, Loh MH, Tan CH. Care of the pregnant woman with coronavirus disease 2019 in labor and delivery: anesthesia, emergency cesarean delivery, differential diagnosis in the acutely ill parturient, care of the newborn, and protection of the healthcare personnel. AJOG. 2019;66-74:e3. 
15. Guascha E, Broglya N, Manriqueb S. Recomendaciones prácticas en la paciente obstétrica con infección por COVID-19. Rev Esp Anestesiol Reanim. 2020;67(8):438-45.
Cite this article as: Thakur S, Kaushal S, Kaur H, Kaundal A. Safe obstetric anesthesia practiceCOVID-19. Int J Reprod Contracept Obstet Gynecol 2021;10:4609-13. 